\title{
Le pharmacien prescripteur : Les vents nous sont-ils favorables?
}

\author{
par Rosemary A Bacovsky
}

$\mathrm{L}$ ors d'une séance de formation sur la responsabilité civile liée à l'élargissement du champ de pratique à Calgary en mars 2012, de nombreux pharmaciens ont vertement critiqué le gouvernement de l'Alberta pour avoir accordé aux pharmaciens, le $1^{\text {er }}$ juillet 2012, des honoraires pour le renouvellement d'ordonnances. Les pharmaciens ne critiquaient pas le montant des honoraires, mais plutôt le fait qu'ils devaient s'acquitter de cette tâche (c.-à-d. renouveler des ordonnances) pour garder tout client qui pourrait demander ce service et aussi le fait que les pharmaciens devraient se conformer aux normes de pratique de cette activité.

Imaginez des pharmaciens prescripteurs, qui de surcroit seraient payés par le gouvernement pour le faire! Ce scénario était inimaginable lorsque j'ai obtenu mon diplôme en 1977. La pharmacie clinique en général et les pharmaciens qui formulaient des recommandations relatives à des changements d'ordonnance étaient alors des concepts émergents. Le pharmacien prescripteur semblait toujours un rêve inatteignable au début des années 2000 alors que moi-même et d'autres pharmaciens de l'Alberta défendaient vigoureusement l'idée d'une loi donnant le droit aux pharmaciens de prescrire de façon indépendante. Les pharmaciens ont prescrit des médicaments de façon subordonnée depuis des dizaines d'années, à la fois de façon officielle (p. ex., par l'intermédiaire de la substitution thérapeutique et d'autres politiques des établissements de santé) et de façon non officielle (p. ex., dans la communauté, par l'intermédiaire d'ententes avec les médecins). J'ai rédigé des documents de référence pour l'Ordre des pharmaciens de l'Alberta, participé à des groupes de travail, fait pression auprès de politiciens et de fonctionnaires, et passé la plus grande partie de mes temps libres à essayer de convaincre mes collègues pharmaciens que cet élargissement du champ de pratique était essentiel pour la croissance de la profession, voire sa survie. Je ne pensais pas que cela se produirait de mon vivant, mais le 1er avril 2007, les pharmaciens de l'Alberta ont obtenu le droit de prescrire des médicaments de façon indépendante. Il s'est écoulé cinq années depuis ce "miracle ", mais très peu de pharmaciens se sont prévalus de ce droit, même si les services sont rémunérés ${ }^{1}$.
En plus d'avoir le droit d'adapter les ordonnances et de prescrire en cas d'urgence, les pharmaciens de l'Alberta peuvent également faire la demande auprès de l'Ordre des pharmaciens de l'Alberta pour obtenir le « droit de prescrire additionnel », qui leur permet d'initier un traitement. Cependant, des quelque 4300 pharmaciens actifs inscrits à l'Ordre des pharmaciens de l'Alberta au 29 février 2012, seulement 155 avaient le droit de prescrire additionnel, la plupart dans la pratique communautaire $^{2}$. Je trouve cela décevant, à la fois en termes de nombre absolu et de nombre relativement faible provenant de la pratique hospitalière. Cet élargissement du champ de pratique aurait été extrêmement utile lorsque j'étais en pratique hospitalière, ce qui était la raison pour laquelle je défendais la cause du droit de prescrire indépendant des pharmaciens.

Le manque d'intérêt actuel des pharmaciens d'hôpitaux ne me surprend pas, car seulement deux des neuf régies régionales de la santé ont approuvé le droit de prescrire des pharmaciens avant la fusion de 2008 qui a créé l'agence provinciale des services de la santé de l'Alberta. Environ à cette époque, j’ai parlé à un pharmacien d'hôpital dont j'admirais la pratique clinique. Il avait alors fait une demande de droit de prescrire additionnel, mais seulement parce que l'hôpital pour lequel il travaillait l'exigeait pour son poste. Le processus de demande le rebutait au plus haut point, car il trouvait cela une perte de temps, vu qu'il s'occupait depuis des années de la prise en charge de la pharmacothérapie, sans cette reconnaissance officielle. Ce qu'il n'a pas su constater, c'est que le statu quo n'aurait pas été conforme à la nouvelle loi. Les services de la santé de l'Alberta appuient le droit de prescrire des pharmaciens et a rendu le droit de prescrire additionnel une exigence pour plusieurs postes, et de nombreux pharmaciens exerçant dans les réseaux de soins de santé primaires et les cliniques de médecine familiale obtiennent maintenant ce droit.

Lorsqu'on examine l'acceptation du droit de prescrire par les pharmaciens, je trouve utile de comparer l'attitude des pharmaciens à celle des infirmières et infirmiers praticiens qui ont obtenu le droit de prescrire, souvent plus tôt que les pharmaciens. Dans la plupart des provinces et territoires, les infirmières et infirmiers praticiens ont un champ de pratique 
plus large que celui des pharmaciens pour ce qui est de la prescription de médicaments. Par exemple, ils auront bientôt le droit de prescrire des stupéfiants et des médicaments contrôlés ${ }^{3}$. Je n'ai lu aucune plainte émanant des infirmières et infirmiers praticiens au sujet de ces élargissements appréhendés de leur champ de pratique. Au contraire, ils semblent accueillir favorablement leurs nouvelles responsabilités. D'ailleurs, l'Ontario a mis en œuvre des cliniques gérées par des infirmières et infirmiers praticiens. De même, l'Alberta a un projet pilote de cliniques de santé familiale pouvant fonctionner sans médecin, où les infirmières et infirmiers praticiens seront probablement les professionnels de la santé clés. Devrions-nous nous inquiéter que les infirmières et infirmiers praticiens puissent jouer le rôle principal dans la prise en charge de la pharmacothérapie sans médecin?

Par contraste, les pharmaciens ont bien accueilli un autre aspect du champ de pratique élargi : l'injection de médicaments. Au 29 février 2012, un total de 1535 pharmaciens en Alberta avaient obtenu le droit d'administrer des médicaments par injection, une augmentation de $35 \%$ par rapport à l'année précédente. En 2011, ces pharmaciens ont participé au programme de vaccination provinciale contre la grippe et ont vacciné plus de 81600 Albertains².

Ce qui s'est produit en Alberta est en train de se "reproduire" ailleurs dans le pays. Présentement, les pharmaciens de toutes les provinces ont le droit de prescrire des médicaments, malgré diverses restrictions. Une législation plus cohérente est requise pour prévenir les trop grandes variations dans le champ de pratique à l'échelle du pays. Cette cohérence peut être facilitée par l'Accord de mobilité à l'intention des pharmaciens canadiens (http://napra.ca/Content_Files/Files/ Mobility_Agreement_FR_signed_final_compressed.pdf) et l'Accord sur le commerce intérieur (http://www.ait-aci.cal index_fr/intro.htm), ce dernier comprenant les principes d'exigences homogènes, et aussi par le désir des gouvernements provinciaux et fédéral d'accroître la mobilité des professionnels de la santé. Les gouvernements veulent également accroître l'accès public à la prise en charge appropriée de la pharmacothérapie, souvent par des prescripteurs autres que des médecins.

La plupart des régimes d'assurance maladie publics et certains privés remboursent les honoraires pour les services de prescription et de gestion des médicaments. Les propriétaires de pharmacie reconnaissent et appuient ces services qui sont une source croissante de revenus. D'autres professionnels de la santé et le grand public pourraient devenir des agents catalyseurs de l'exercice du droit de prescrire par les pharmaciens, à mesure que ces groupes s'attendent et exigent des pharmaciens qu'ils non seulement renouvellent leurs ordonnances, mais prennent en charge leurs pharmacothérapies.
Je reconnais que de nombreux pharmaciens sont satisfaits du statu quo et ne prescriront que s'ils y sont contraints. Mais les vents nous sont favorables, tout comme ils l'étaient pour l'adoption de la pharmacie clinique lorsque j'ai reçu mon diplôme il y a 35 ans. Les 155 pharmaciens de l'Alberta ayant obtenu le droit de prescrire additionnel et d'autres pharmaciens d'ailleurs au Canada qui ont embrassé le droit de prescrire et la prise en charge de la pharmacothérapie peuvent être des modèles et une source d'inspiration pour tous les pharmaciens. Ils mettent de l'avant les modèles de pratique et les données probantes qui permettront un jour à tous les pharmaciens d'avoir le droit de prescrire indépendamment, dont le droit d'initier un traitement, et d'utiliser de façon habituelle et proactive leurs connaissances et leur droit de prescrire. J'espère que cela se produira aussi de mon vivant.

[Traduction par l'éditeur]

\section{Références}

1. Law M, Ma T, Fisher J, Sketris IS. Independent pharmacist prescribing in Canada. Can Pharm J 2012;145(1):17-23.

2. 2011-2012 annual report. Edmonton (AB): Alberta College of Pharmacists; 2012. Publié au https://pharmacists.ab.ca/Content_Files/Files/AR2011_ 12.pdf. Consulté le 23 octobre 2012.

3. Le gouvernement Harper veut permettre à plus de professionnels de la santé de prescrire des substances licites contrôlées [communiqué de presse]. Ottawa (ON): Santé Canada; 13 mai 2012. Publié au www.hc-sc.gc.ca/ ahc-asc/media/nr-cp/_2012/2012-71-fra.php. Consulté le 23 octobre 2012.

Rosemary A Bacovsky, B. Sc. Pharm., M. Pharm., MHSA, FASHP, FCSHP, est présidente d'Integra Consulting Ltd à Calgary, en Alberta. Elle a reçu le Prix pour service distingué de la Société canadienne des pharmaciens d'hôpitaux en 1997.

\section{Adresse de correspondance :}

Rosemary A Bacovsky

Integra Consulting Ltd

38201 Street SW

Calgary AB T2S 1 R3

Courriel : bacovsky@telusplanet.net

Cet article est le premier d'une série spéciale d'éditoriaux, sous la coordination de William McLean, Pharm. D., FASHP, FCCP, et du Comité de rédaction du JCPH, rédigés par des récipiendaires du Prix pour service distingué de la SCPH qui sont invités à commenter une question d'actualité pour la profession. L'éditorialiste invité doit fournir un bref point de vue sur l'évolution de cet aspect particulier au cours de sa carrière et commenter sa vision de l'évolution future de cet aspect. Par conséquent, l'éditorialiste invité combinera une perspective historique et future de même que des éléments de réflexion. 